\title{
Myosin II isoforms identify distinct functional modules that support integrity of the epithelial zonula adherens
}

\author{
Michael Smutny ${ }^{1}$, Hayley L. Cox ${ }^{1}$, Joanne M. Leerberg ${ }^{1}$, Eva M. Kovacs ${ }^{1}$, Mary Anne \\ Conti $^{2}$, Charles Ferguson ${ }^{1}$, Nicholas A. Hamilton ${ }^{1}$, Robert G. Parton ${ }^{1}$, Robert S. Adelstein ${ }^{2}$, \\ and Alpha S. Yap ${ }^{1,3}$ \\ ${ }^{1}$ Institute for Molecular Bioscience, Division of Molecular Cell Biology, The University of \\ Queensland, St. Lucia, Brisbane, Queensland 4072, Australia \\ ${ }^{2}$ Laboratory of Molecular Cardiology, National Heart, Lung and Blood Institute, National Institutes \\ of Health, Bethesda, Maryland 20892-1583, USA
}

\begin{abstract}
Classic cadherin receptors cooperate with regulators of the actin cytoskeleton to control tissue organization in health and disease. At the apical junctions of epithelial cells, the cadherin ring of the zonula adherens (ZA) couples with a contiguous ring of actin filaments ${ }^{1-3}$ to support morphogenetic processes such as tissue integration and cellular morphology $y^{4,5}$. However, the molecular mechanisms that coordinate adhesion and cytoskeleton at these junctions are poorly understood. Previously we identified non-muscle myosin II as a target of Rho signalling that supports cadherin junctions in mammalian epithelial cells ${ }^{6}$. Myosin II has various cellular functions, which are increasingly attributable to the specific biophysical properties and regulation of its different isoforms ${ }^{7}$. Here we report that myosin II isoforms have distinct and necessary roles at cadherin junctions. Although two of the three mammalian myosin II isoforms are found at the ZA, their localization is regulated by different upstream signalling pathways. Junctional localization of myosin IIA required E-cadherin adhesion, Rho/ROCK and myosin light-chain kinase, whereas junctional myosin IIB depended on Rap1. Further, these myosin II isoforms support E-cadherin junction integrity by different mechanisms. Myosin IIA RNA-mediated interference (RNAi) selectively perturbed the accumulation of E-cadherin in the apical ZA, decreased cadherin homophilic adhesion and disrupted cadherin clustering. In contrast, myosin IIB RNAi decreased filament content, altered dynamics, and increased the lateral movement of the perijunctional actin ring. Myosin IIA and IIB therefore identify two distinct functional modules, with different upstream signals that control junctional localization, and distinct functional effects. We propose that these two isoform-based modules cooperate to coordinate adhesion receptor and F-actin organization to form apical cadherin junctions.
\end{abstract}

Three myosin II isoforms are expressed in mammalian cells ${ }^{8,9}$; all were identified by mass spectrometry in MCF7 breast epithelial cells, although in different proportions (myosin IIA,

\footnotetext{
(C) 2010 Macmillan Publishers Limited. All rights reserved.

${ }^{3}$ Correspondence should be addressed to A.Y. (a.yap@uq.edu.au).

Note: Supplementary Information is available on the Nature Cell Biology website.
}

\section{AUTHOR CONTRIBUTIONS}

M.S. and A.S.Y. conceived and designed the experiments. M.S., H.L.C., E.M.K., M.A.C. and C.F. performed experiments and data analysis. J.M.L. and N.A.H. contributed to data analysis. R.G.P., E.M.K. and R.S.A. contributed to discussions and intellectual input. M.S. and A.S.Y. wrote the paper. 
$54.8 \pm 4.2 \%$; myosin IIB, $12.5 \pm 2.9 \%$; myosin IIC, $32.5 \pm 5.4 \%$ (means \pm s.e.m.); see Methods). However, only myosin IIA and myosin IIB were found at cell-cell contacts, notably in cortical rings at the apical poles of confluent MCF7 cells, contiguous with the junctional rings of E-cadherin (Fig. 1a). Moreover, whereas segregation of myosin IIA and IIB is seen in other contexts ${ }^{10}$, direct comparison of their localization using antibodies from different species confirmed that both isoforms co-localized in apical rings (Fig. 1b). Myosin IIA and IIB also decorated the apical ring of F-actin and were in close proximity to the tight junctions (Supplementary Information, Fig. S1).

We found previously that the cortical localization of myosin II responded to E-cadherin adhesion and cell signalling 6 , but we did not examine their influence on different myosin isoforms. Accordingly, we first tested how E-cadherin short interfering RNA (siRNA) (Fig. 1c; Supplementary Information, Fig. S2a) affected the localization of myosin II isoforms. Total cellular levels of myosin IIA and IIB were unaffected by E-cadherin knockdown (KD; not shown). However, myosin IIA staining at cell-cell contacts (identified by ZO-1;

Supplementary Information, Fig. S2b) was decreased by about $50 \%$ after cadherin depletion, whereas junctional myosin IIB localization was unaffected (Fig. 1d, e; Supplementary Information, Fig. S2b). We also acutely perturbed cadherin function with a functionblocking monoclonal antibody (SHE78-7) ${ }^{11,12}$. A short exposure to SHE78-7 preserved the overt integrity of cadherin contacts but decreased the level of myosin IIA at the junctional region without affecting that of myosin IIB (Supplementary Information, Fig. S2c, d). This implied that E-cadherin adhesion was necessary for the junctional localization of myosin IIA but not for that of myosin IIB.

Similarly, the junctional localization of these myosin II isoforms was differentially sensitive to the Rho-ROCK signalling pathway, a well-characterized upstream activator of myosin II. The level of junctional myosin IIA was decreased when Rho was inhibited with cellpermeant C3-transferase (Fig. 2a, b) or when ROCK was blocked with Y-27632 (Fig. 2a, c); however, junctional myosin IIB was not affected by either treatment (Fig. 2a-c).

One way that the Rho-ROCK pathway regulates myosin II is by controlling the phosphorylation status of the regulatory light chain to promote the ATPase activity of $\operatorname{myosin}^{7,9}$. Myosin light-chain kinase (MLCK) is a major regulatory light-chain kinase that also influences the cortical localization of myosin II (ref. 13). We found that the MLCK inhibitor ML7 decreased the level of junctional myosin IIA without affecting that of junctional myosin IIB (Fig. 2d; Supplementary Information, Fig. S3a). Overall, these findings suggested that activation was necessary for myosin IIA to localize to junctions, but not for myosin IIB to do so. To test this hypothesis further we treated cells with blebbistatin, which directly blocks myosin II in a products complex with a low affinity for actin ${ }^{14}$. The level of junctional myosin IIA was decreased by blebbistatin, whereas myosin IIB persisted at contacts (Fig. 2e) despite some local redistribution towards tricellular junctions (Supplementary Information, Fig. S3a). Taken together, these data suggest that motor activation is necessary for the junctional localization of myosin IIA, whereas the localization of myosin IIB responds to a different pathway.

In efforts to identify upstream regulators of myosin IIB localization, we then examined the effect of Rap1, which can support ZA integrity ${ }^{15}$ and influences myosin II in Dictyostelium and Drosophila ${ }^{16,17}$. Indeed, siRNA directed against Rap1A (Supplementary Information, Fig. S3b) decreased the level of junctional myosin IIB by about $50 \%$ but did not affect that of myosin IIA (Fig. 2f, g). Total cellular levels of these myosin isoforms were unchanged (Supplementary Information, Fig. S2b), indicating that Rap1A influences the subcellular localization of myosin IIB. Overall, we conclude that, although both myosin IIA and IIB accumulate at the perijunctional apical ring, each localizes there in response to different 
upstream signals. We emphasize that our data do not exclude a role for Rho/ROCK and MLCK in controlling the motor activity of myosin IIB, but they indicate that these signals do not determine the junctional localization of this isoform.

We then selectively depleted each isoform with lentiviral shRNA (Fig. 3a; Supplementary Information, Fig. S4a) to test their junctional impact. Myosin IIA depletion markedly altered the distribution of E-cadherin. The prominent apical ring of E-cadherin seen in control cells was replaced by numerous clusters distributed generally throughout the lateral surfaces (Fig. 3b). Further, many contacts between myosin IIA KD cells appeared flattened (en face) compared with control contacts when imaged by both fluorescence (Fig. 3b) and electron microscopy (not shown). Quantification of the lateral distribution of cadherin fluorescence across the cell-cell contacts confirmed that, whereas control contacts showed a sharp, narrow peak of fluorescence (Fig. 3c-e; Supplementary Information, Fig. S4c) that corresponded to the apical junctions, cadherin fluorescence in myosin IIA KD cells was distributed more widely (Fig. 3c, d), with a lower peak intensity (Fig. 3c, e). Total cellular and surface levels of E-cadherin were not altered in myosin IIA KD cells (Supplementary Information, Fig. S4b), reinforcing the interpretation that cadherin remained at the lateral surfaces but failed to concentrate in apical junctional rings.

Depletion of myosin IIB also affected the integrity of cadherin organization at apical cellcell contacts, with numerous breaks in the linear continuity of the ZA (Fig. 3b). However, in contrast to myosin IIA KD, E-cadherin still stained intensely at the apical interfaces between cells, with only a slight decrease in peak E-cadherin intensity and a narrow distribution of staining that was indistinguishable from that of control contacts (Fig. 3c-e; Supplementary Information, Fig. S4c). This indicated that the ability of cells to concentrate E-cadherin at the apices of cell-cell contacts was preserved despite myosin IIB KD. Moreover, neither total nor surface levels of E-cadherin were altered by myosin IIB depletion (Supplementary Information, Fig. S4b). These findings demonstrate that myosin IIA and myosin IIB support the integrity of the ZA ring in different ways. Myosin IIA is necessary for E-cadherin to concentrate apically in the ZA, whereas in myosin IIB KD cells cadherin can concentrate but the ZA becomes fragmented, perhaps in response to orthogonal forces acting on the cadherin junctions. The impact of double myosin IIA/IIB KD resembled that of myosin IIA depletion, with the apical cadherin ring being replaced by puncta of cadherin distributed more generally at the interfaces between cells (Supplementary Information, Fig. S4d). The cadherin ZA also seemed to depend on these myosin isoforms more than the tight junctions did. ZO-1 staining remained largely intact on depletion of either myosin II isoform (Supplementary Information, Fig. S4a), although it was disrupted by double myosin KD (Supplementary Information, Fig. S4d).

We then sought to examine which intrinsic properties were necessary for these motors to support the ZA. All myosin II isoforms possess both the ability to bind F-actin and to slide filaments by their motor capacity ${ }^{7}$. Mutants that selectively uncouple these features have provided evidence that both these properties can make distinct contributions to myosin's cellular functions ${ }^{9,18-20}$. Accordingly, we expressed mutants of either myosin IIA (N93K) and myosin IIB (R709C), which bound F-actin but were ineffective filament-sliders, in the appropriate KD background to test the molecular contributions of filament binding and sliding to ZA integrity ${ }^{21,22}$.

Expression of RNAi-resistant wild-type myosin IIA restored the concentrated apical cadherin rings in myosin IIA KD cells, confirming that the impact of RNAi on cadherin distribution was specific for depletion of this isoform (Fig. 3f). Strikingly, expression of N93K myosin IIA restored the apical cadherin ring almost totally (Fig. 3f). Similarly, expression of myosin IIB rescued the myosin IIA KD phenotype (Fig. 3f). Expression of 
wild-type myosin IIB also restored ZA integrity in myosin IIB KD cells (Fig. 3g). However, the R709C mutant of myosin IIB was conspicuously less effective in restoring ZA integrity (Fig. 3g); nor could exogenous myosin IIA rescue the myosin IIB phenotype (Fig. 3g). Therefore, although actin binding is sufficient for myosin IIA to maintain the apical cadherin ring, contractility (both filament binding and sliding) is essential for myosin IIB to have its junctional effect. Our experience with myosin IIA is consistent with increasing evidence that anchorage through actin-filament binding may dominate the contribution of myosin II isoforms in a variety of adhesive contexts ${ }^{18,20}$. Yet MLCK and Rho/ROCK signalling activates both features of myosin IIA. How, then, might filament binding come to dominate at the ZA? One possibility is that resistance transmitted from cadherin receptors at the ZA leads to very slowly cycling cross-bridges of myosin IIA, decreasing filament sliding but leaving the motor anchored to F-actin ${ }^{7}$.

These differences may ultimately reflect the intrinsic motor properties of these two myosin isoforms. Myosin IIB has a significantly longer duty ratio than myosin IIA, a property that would confer a greater ability to generate sustained contractile force ${ }^{7}$. One implication is that myosin IIB might be the dominant contractile force-generator at junctions in established epithelial monolayers. Consistent with this, myosin IIB KD decreased apparent apicolateral tension, reflected in a loss of taut linear ZO-1 staining ${ }^{23}$, whereas myosin IIA KD had no similar effect (Supplementary Information, Fig. S4a). A lower capacity for sustained contractility also potentially explains why myosin IIA could not restore the myosin IIB KD phenotype.

How, then, might these myosin isoforms determine the integrity of the ZA? Strong adhesion, driven by lateral clustering of cadherins ${ }^{24}$, is commonly thought to be the raison d'être of the ZA. We therefore examined the impact of myosin IIA and IIB on these properties of cadherin function (Fig. 4a). We assessed cadherin-specific adhesion by using laminar flow assays measuring cellular resistance to detachment from substrata coated with functional cadherin ligands $(\mathrm{hE} / \mathrm{Fc})^{11,25}$. Cadherin homophilic adhesion was reduced by about $25 \%$ in myosin IIA KD cells (Fig. 4a), which is consistent with our earlier observation that adhesion was decreased by blebbistatin, Y27632 or ML-7 (ref. 6). However, homophilic adhesion was not affected by myosin IIB depletion. We then assessed lateral clustering 24,26 by examining the distribution of cellular cadherins when cells adhered to $\mathrm{hE} / \mathrm{Fc}$-coated substrata, which specifically induce the lateral reorganization of cadherins into clusters ${ }^{6,27,28}$. We typically observed two patterns of clusters: small dot-like puncta and larger streaks (macroclusters) (Fig. 4b; refs 6, 27, 28). Both the frequency and length of macroclusters were substantially decreased in myosin IIA KD cells compared with either myosin IIB KD or control cells (Fig. 4c, d), which is consistent with the selective impact of myosin IIA KD on homophilic adhesion (Fig. 4a). Despite an unchanged cluster number (Fig. 4c), macrocluster length was increased by myosin IIB depletion (Fig. 4d), suggesting that although this isoform is not necessary for clustering, it may affect cluster turnover. Overall, we propose that myosin IIA functions as a cortical organizer to promote large-scale clustering and adhesive strengthening of E-cadherin, thereby supporting cadherin accumulation in the apical ring of the ZA.

Given its lack of demonstrable effects on E-cadherin, we then considered how myosin IIB might contribute to apical junction integrity. Because blebbistatin decreases the density of the perijunctional actin ring ${ }^{6}$, we examined the impact of each isoform on F-actin content in the apical junctional region. Myosin IIB depletion decreased the intensity of phalloidin staining in the apical actin ring (Fig. 5a-c; Supplementary Information, Figs S5 and S6a), but myosin IIA KD had no detectable effect. The residual F-actin was often found between fragmented E-cadherin contacts in the myosin IIB KD cells, whereas the scant remaining 
apical cadherin overlapped the unchanged apical actin rings in myosin IIA KD cells (Supplementary Information, Fig. S5).

We then assessed junctional actin dynamics by fluorescence recovery after photobleaching (FRAP) of green fluorescent protein (GFP)-tagged actin. Junctional actin recovery was unaffected by myosin IIA KD, but myosin IIB KD decreased the proportion of actin undergoing exchange in the apical ring (Fig. 5d; Supplementary Information, Fig. S6b and Table S1). This relative decrease in labile actin might reflect an overall stabilization of junctional F-actin or alternatively the decreased size of a dynamic subpopulation of actin filaments. Either interpretation implies that myosin IIB maintains dynamic actin filaments in the apical ring. Kymographic analysis further revealed that the apical actin ring undergoes greater movement orthogonal to the junction in myosin IIB KD cells than in either control or myosin IIA KD cells, where the rings are relatively static (Fig. 5e). Overall, these findings strongly suggest that myosin IIB supports dynamic actin filaments in the apical ring and allows it to resist orthogonal forces that would act to disrupt the E-cadherin ZA. The observed requirement for myosin IIB contractility may then reflect a contribution of lateral tension to physical reinforcement of the apical ring.

It is increasingly apparent that the ZA is the product of multiple cellular processes that functionally and physically link adhesion to the cytoskeleton ${ }^{29,30}$. ZA integrity requires mechanisms to concentrate cadherin adhesion receptors at the apico-lateral interface between cells, and mechanically reinforce these junctions to resist disruptive forces that play on contacts. Our new findings demonstrate that the contribution of myosin II to ZA integrity actually reflects the coordinated action of two distinct processes, identified by separate myosin II isoforms. Myosin IIA seems to function as a cortical organizer to concentrate Ecadherin in the ZA, whereas myosin IIB supports integrity of the apical actin ring to prevent fragmentation of the ZA, perhaps by reinforcing it to resist disruptive orthogonal forces. These functional differences, together with evidence that their junctional localization is regulated by distinct upstream signalling pathways, identify these myosins as parts of distinct functional modules. How these myosin II-based modules are coordinated at the ZA is now a question for further investigation.

\section{METHODS}

\section{Cell culture, transfections and hE/Fc-based assays}

MCF-7 cells and Chinese hamster ovary (CHO) cells have been described previously ${ }^{31,32}$. HEK-293T cells were cultured in DMEM complete growth medium. Cells were transfected with Lipofectamine 2000 (Invitrogen) in accordance with the manufacturer's instructions, and then analysed $24-72 \mathrm{~h}$ after transfection.

$\mathrm{hE} / \mathrm{Fc}$ preparation and assays were described previously 24,33 . Adhesion to $\mathrm{hE} / \mathrm{Fc}$ requires a cellular cadherin, being absent in $\mathrm{CHO}$ cells lacking E-cadherin, and is blocked by chelation of extracellular $\mathrm{Ca}^{2+}$ and by SHE78-7, a function-blocking antibody directed against the Ecadherin ectodomain ${ }^{33}$. Surface expression of E-cadherin was measured by the sensitivity to surface trypsinization as described previously ${ }^{34}$.

\section{Plasmids, cloning and in vitro mutagenesis}

pEGFP-NMHC-2A N93K and pEGFP-NMHC-2B R709C have been described previously ${ }^{21,22}$. NMHC-2A and NMHC-2B RNAi-resistant constructs were generated by mutating two bases in each siRNA target sequence by site-directed mutagenesis (QuickExchange kit; Fermentas) and confirmed by sequencing. Underlined bases in NMHC-2A (5'-GGCCAA $\underline{G}$ GAGATGAGAAG-3') and NMHC-2B (5' - 
GGAAGGATGTAGACCGTAT-3') indicate mutations in RNAi-targeted sequences. For live-cell imaging we used a pEGFP-actin vector from Clontech.

\section{Mass spectrometry}

Cell culture plates (10 cm in diameter) of MCF-7 cells were scraped and cells were extracted with RIPA buffer (Santa Cruz Biotechnology) modified by the addition of $130 \mathrm{mM} \mathrm{NaCl}$ (250 mM NaCl total), $5 \mathrm{mM}$ EGTA, $0.1 \mathrm{mM}$ phenylmethylsulphonyl fluoride, $10 \mu \mathrm{g} \mathrm{ml}^{-1}$ leupeptin, protease inhibitor cocktail (Sigma), $1 \mathrm{mM}$ dithiothreitol and $5 \mathrm{mM} \mathrm{MgATP}{ }^{2-}$. After $10 \mathrm{~min}$ on ice, the samples were centrifuged at $10,000 \mathrm{~g}$ for $10 \mathrm{~min}$, and the supernatant was used for SDS-PAGE. The Coomassie blue-stained bands near the 205-kDa molecular size marker were excised, destained, reduced and alkylated, digested with trypsin and submitted to the NHLBI Proteomics Core Facility for analysis by liquid chromatography-tandem mass spectrometry. Peptide numbers for each of the NMHC II isoforms were counted and compared with total NMHC II peptides; the standard deviation of measurements was calculated.

\section{Antibodies}

Primary antibodies used in this study were as follows: mouse monoclonal antibody (mAb) against the cytoplasmic domain of E-cadherin (Transduction Laboratories); mouse mAb HECD-1 against the ectodomain of E-cadherin (a gift from Peggy Wheelock, University of Nebraska, Omaha, NE; with the permission of M. Takeichi); rabbit polyclonal antibody (pAb) for non-muscle myosin IIA heavy chain (Covance); rabbit pAb for non-muscle myosin IIB heavy chain (Covance); mouse mAb CMII 25 against myosin IIB heavy chain (DSHB, The University of Iowa); rabbit pAb or mouse mAb against GFP (Molecular Probes/Invitrogen); rabbit pAb and mouse mAb against human ZO-1 (Zymed Laboratories); mouse $\mathrm{mAb}$ against human Rap1 (BD Biosciences); the E-cadherin-blocking antibody SHE78-7 (Zymed Laboratories) directed against the E-cadherin ectodomain, used on MCF-7 monolayers at a dilution of 1:50 for $1 \mathrm{~h}$. F-actin was stained with AlexaFluor 488-phalloidin or 594-phalloidin (1:1,000 dilution; Invitrogen). Secondary antibodies were species-specific antibodies conjugated with AlexaFluor 488, 594 or 647 (Invitrogen) for immunofluorescence, or with horseradish peroxidase (Bio-Rad Laboratories) for immunoblotting.

\section{Immunofluorescence microscopy and image analysis}

Cells were fixed either with methanol on ice for 5 min or with $4 \%$ paraformaldehyde in cytoskeleton stabilization buffer (10 mM PIPES pH 6.8, $100 \mathrm{mM} \mathrm{KCl,} 300 \mathrm{mM}$ sucrose, 2 mM EGTA, $2 \mathrm{mM} \mathrm{MgCl}_{2}$ ) at $22^{\circ} \mathrm{C}$ for $20 \mathrm{~min}$ and subsequently permeabilized with $0.25 \%$ Triton-X in PBS for 4 min at room temperature.

Images were acquired with an IX81 Olympus microscope (60' and $100^{\prime} 1.4$ numerical aperture objectives) and a Hamamatsu Orca-1 ER camera for epi-illumination driven by Metamorph imaging software (version 7; Universal Imaging). Confocal images were captured with a Zeiss 510 Meta laser-scanning confocal microscope, and $z$-stacks were processed with the LSM510 software.

Quantitative analysis of fluorescence intensity measurements of myosin II (Figs 1 and 2) was derived from three independent experiments and performed with the use of ImageJ. In brief, contacts were selected at random and a region of interest (ROI) was defined that covered the whole contact (vertex to vertex). Background was removed as follows. The image was duplicated and a mask was created from one by applying a threshold. Multiplying the original image by the mask image using the calculator Plus plugin then gave an image for which all background pixels were set to intensity 0 . Total pixel intensity values of 
myosin II at cell junctions were taken from the histogram function. and average pixel intensities were calculated therefrom. Graphs were plotted and analysed using Prism 5 software.

Quantitative analysis of E-cadherin and F-actin at contacts (Figs 3c, d and 5a, b) was performed in ImageJ with the line scan function. Within ImageJ, a line of length 63 pixels was selected centred on, and perpendicular to, randomly chosen contacts. The PlotProfile feature of ImageJ was used to record the pixel intensities along the selected line, and the results were saved in text format. The process was repeated for 24 contacts derived from three independent experiments. The data for each profile were then imported into Prism 5. Pixel intensity of background regions in the microscopy imaging was typically non-zero and was corrected for by subtracting a constant value from each of the intensity profiles. This led to each profile being approximately Gaussian in shape, with the tails going to zero. Using Prism 5, a Gaussian curve was fitted to each (background-corrected) intensity profile, and the standard deviation of the fitted curve was recorded. The full width at half-maximum (FWHM) was then calculated for each curve as the standard deviation multiplied by 2.3548 . For a given experiment the mean and the standard deviation of the FWHM were calculated. Similarly, the mean and standard deviation of the maximum heights of the fitted curves were calculated.

Quantitative analysis of cadherin junction rescue (Fig. 3f, g) was performed by imaging the apical distribution of E-cadherin and comparing the morphology of E-cadherin at contacts with that in control cells. Cells that possessed contiguous ring-like E-cadherin staining at the apical (ZA) region were defined as 'intact'. To account for variability we quantified 90 randomly chosen contacts from each rescue experiment. Relative proportions of intact ZA were plotted in bar graphs in which 1 represented $100 \%$ restoration.

\section{Live-cell imaging and FRAP analysis}

Cells were grown on glass-bottomed dishes (MaTek) and live-cell imaging was performed with a Zeiss 510-Meta laser-scanning confocal microscope with a heated stage maintained at $37^{\circ} \mathrm{C}$. During imaging, cells were incubated in Hanks balanced salt solution without phenol red supplemented with $10 \mathrm{mM}$ HEPES $\mathrm{pH} 7.4$ conatining $5 \mathrm{mM} \mathrm{CaCl}_{2}$. For fluorescence time-lapse analysis in MCF-7 cells we imaged transiently transfected GFP-actin $24 \mathrm{~h}$ after transfection at cell-cell contacts with a 100' 1.4 numerical aperture oil-immersion lens at ' 8 magnification. Time-lapse images were acquired before and after photobleaching at $2.69 \mathrm{~s}$ per frame for a total time of $3 \mathrm{~min}$. For FRAP analysis, GFP-actin was photobleached with a constant ROI bleaching area for all experiments with $100 \% 488 \mathrm{~nm}$ laser transmission resulting in maximum bleach of about $50 \%$ of the GFP-actin at contacts, and fluorescence recovery was observed to steady-state levels. We measured eight FRAP profiles of GFPactin in control, myosin IIA and myosin IIB knockdown cells from four individual experiments, and data were analysed in ImageJ as follows: for better visualization of junctional GFP-actin, we used the median plug-in of ImageJ; we used the TurboReg plug-in to correct for the lateral movement of GFP-actin. To calculate FRAP profiles we marked a ROI at the bleached GFP-actin area and used the FRAP Profiler plug-in from ImageJ to obtain fluorescence intensity profiles. Fluorescence intensities were corrected for acquisition photobleaching and normalized to the pre-bleach values. Normalized FRAP curves were imported into Prism (GraphPad) and fitted with a recovery curve to calculate the half-times of fluorescence recovery and the plateau levels.

Kymographs were generated in ImageJ by defining a line scan perpendicular to the membrane where GFP-actin was bleached and using the kymograph plug-in. Lateral movement of GFP-actin in the kymographs was calculated by measuring the angle between the position of GFP-actin at the beginning and at the end of the time series. Negative angles 
were normalized to positive ones. Mean angle values in degrees are shown in scatter plot graphs.

\section{siRNA and shRNA knockdowns}

E-cadherin was silenced with Dharmacon SMARTpool siRNA $(50 \mathrm{nM})$ directed against human CDH1 (NM_004360). RAP1A was knocked down with Dharmacon SMARTpool siRNA (100 nM) directed against human RAP1A (NM_002884). Non-targeting siRNA pools were used as controls (Dharmacon). The algorithms from OligoEngine and Dharmacon were used to predict sequences that would lead to silencing of human MYH9 (NM_002473) and MYH10 (NM_005964), respectively. MYH9 silencing sequence, 5' GGCCAAAGAGAACGAGAAG-3'; MYH10 silencing sequence, $5^{\prime}$ GGAAAGATGTGGACCGTAT- $3^{\prime}$. Predicted sequences were used to design shRNAs containing a stem loop sequence based on previous studies ${ }^{35}$, and these were cloned into the lentivirus expression vector LentiLox pLL5.0 (refs 36, 37). In brief, shRNA was cloned downstream of the U6 promoter ( $\mathrm{HpaI}$ and $\mathrm{Xhol}$ ) into a lentivirus expression vector pLL5.0 (backbone pLL3.7) carrying a soluble EGFP as reporter gene. A modified version of pLL5.0 was constructed by replacing the EGFP marker with a cassette expressing mCherry.

The pLL5.0 and packaging vectors were transfected into HEK-293T cells by $\mathrm{CaCl}_{2}$ precipitation. At $16 \mathrm{~h}$ after transfection, cells were treated with sodium butyrate $(10 \mathrm{mM})$ to increase gene induction. Virus-like particles (VLPs) were harvested from the supernatant 48-72 $\mathrm{h}$ after transfection, and virus was concentrated on poly(vinylidene difluoride) spin columns (Millipore) to obtain high titres of VLP fractions. Aliquots of virus were subsequently used for titration or stored at $-80{ }^{\circ} \mathrm{C}$. Titres were determined by infecting HEK-293T cells with serial dilutions of concentrated lentivirus. We determined GFP or mCherry expression of infected cells by flow cytometry $48-72 \mathrm{~h}$ after infection. A typical preparation yielded approximately $10^{8}-5^{\prime} 10^{8}$ plaque-forming units $\mathrm{ml}^{-1}$.

MCF-7 cells were infected with lentiviral particles at a multiplicity of infection of 10 per cell. Cells were incubated at $37{ }^{\circ} \mathrm{C}$ with lentivirus in DMEM complete and Polybrene solution $\left(8 \mu \mathrm{g} \mathrm{ml}^{-1}\right)$ and harvested by trypsinization three days after infection. Single-cell suspensions were sorted by flow cytometry according to moderate to high levels of reporter gene expression. Cells maintained silencing of NMHC-2A and NMHC-2B on protein levels up to 20 days after infection (data not shown).

\section{Supplementary Material}

Refer to Web version on PubMed Central for supplementary material.

\section{Acknowledgments}

We thank our colleagues who provided reagents, especially Jim Bear for the pLL5.0 lentiviral construct, Rachel Murphy and Nigel McMillan for training us in lentiviral work, Regine Hartmann for her assistance with cloning, Suzie Verma and Carmen Buttery for assistance with tissue culture, and our colleagues in the laboratory for their untiring support and encouragement. The work in Australia was funded by the National Health and Medical Research Council (NHMRC) of Australia; that in the USA was supported by the National Institutes of Health (NIH). Confocal microscopy was performed at the Australian Cancer Research Foundation (ACRF) Cancer Biology Imaging Centre at the Institute for Molecular Bioscience, established with the generous support of the ACRF. We gratefully acknowledge the help and advice of Guanghui Wang and the NIH National Heart, Lung and Blood Institute Proteomics Core Facility. M.S. was an Erwin Schroedinger postdoctoral fellow of the Austrian Science Fund (FWF), and R.G.P. and A.S.Y. are Research Fellows of the NHMRC. 


\section{References}

1. Boller K, Vestweber D, Kemler R. Cell-adhesion molecule uvomorulin is localized in the intermediate junctions of adult intestinal epithelial cells. J. Cell Biol. 1985; 100:327-332. [PubMed: 3880756]

2. Hirano S, Nose A, Hatta K, Kawakami A, Takeichi M. Calcium-dependent cell-cell adhesion molecules (cadherins): subclass specificities and possible involvement of actin bundles. J. Cell Biol. 1987; 105:2501-2510. [PubMed: 3320048]

3. Yonemura S, Itoh M, Nagafuchi A, Tsukita S. Cell-to-cell adherens junction formation and actin filament organization: similarities and differences between non-polarized fibroblasts and polarized epithelial cells. J. Cell Sci. 1995; 108:127-142. [PubMed: 7738090]

4. Sawyer JK, Harris NJ, Slep KC, Gaul U, Peifer M. The Drosophila afadin homologue Canoe regulates linkage of the actin cytoskeleton to adherens junctions during apical constriction. J. Cell Biol. 2009; 186:57-73. [PubMed: 19596848]

5. Vaezi A, Bauer C, Vasioukhin V, Fuchs E. Actin cable dynamics and Rho/ROCK orchestrate a polarized cytoskeletal architecture in the early steps of assembling a stratified epithelium. Dev. Cell. 2002; 3:367-381. [PubMed: 12361600]

6. Shewan AM, et al. Myosin 2 Is a key Rho kinase target necessary for the local concentration of Ecadherin at cell-cell contacts. Mol. Biol. Cell. 2005; 16:4531-4532. [PubMed: 16030252]

7. De la Cruz EM, Ostap EM. Relating biochemistry and function in the myosin superfamily. Curr. Opin. Cell Biol. 2004; 16:61-67. [PubMed: 15037306]

8. Vicente-Manzanares M, Ma X, Adelstein RS, Horwitz AR. Non-muscle myosin II takes centre stage in cell adhesion and migration. Nat. Rev. Mol. Cell Biol. 2009; 10:778-790. [PubMed: 19851336]

9. Conti MA, Adelstein RS. Nonmuscle myosin II moves in new directions. J. Cell Sci. 2008; 121:1118. [PubMed: 18096687]

10. Kolega J. Cytoplasmic dynamics of myosin IIA and IIB: spatial 'sorting' of isoforms in locomoting cells. J. Cell Sci. 1998; 111:2085-2095. [PubMed: 9664030]

11. Maddugoda MP, Crampton MS, Shewan AM, Yap AS. Myosin VI and vinculin cooperate during the morphogenesis of cadherin cell-cell contacts in mammalian epithelial cells. J. Cell Biol. 2007; 178:529-540. [PubMed: 17664339]

12. den Elzen N, Buttery CV, Maddugoda MP, Ren G, Yap AS. Cadherin adhesion receptors orient the mitotic spindle during symmetric cell division in mammalian epithelia. Mol. Biol. Cell. 2009; 20:3740-3750. [PubMed: 19553471]

13. Matsumura F. Regulation of myosin II during cytokinesis in higher eukaryotes. Trends Cell Biol. 2005; 15:371-377. [PubMed: 15935670]

14. Kovacs M, Toth J, Hetenyi C, Malnasi-Csizmadia A, Sellers JR. Mechanism of blebbistatin inhibition of myosin II. J. Biol. Chem. 2004; 279:35557-35563. [PubMed: 15205456]

15. Dube N, et al. The RapGEF PDZ-GEF2 is required for maturation of cell-cell junctions. Cell Signal. 2008; 20:1608-1615. [PubMed: 18585005]

16. Boettner B, Van Aelst L. The Rap GTPase activator Drosophila PDZ-GEF regulates cell shape in epithelial migration and morphogenesis. Mol. Cell. Biol. 2007; 27:7966-7980. [PubMed: 17846121]

17. Jeon TJ, Lee DJ, Merlot S, Weeks G, Firtel RA. Rap1 controls cell adhesion and cell motility through the regulation of myosin II. J. Cell Biol. 2007; 176:1021-1033. [PubMed: 17371831]

18. Choi CK, et al. Actin and a-actinin orchestrate the assembly and maturation of nascent adhesions in a myosin II motor-independent manner. Nat. Cell Biol. 2008; 10:1039-1050. [PubMed: 19160484]

19. Conti MA, Even-Ram S, Liu C, Yamada KM, Adelstein RS. Defects in cell adhesion and the visceral endoderm following ablation of nonmuscle myosin heavy chain II-A in mice. J. Biol. Chem. 2004; 279:41263-41266. [PubMed: 15292239]

20. Ma X, Bao J, Adelstein RS. Loss of cell adhesion causes hydrocephalus in nonmuscel myosin II-Bablated and mutated mice. Mol. Biol. Cell. 2007; 18:2305-2312. [PubMed: 17429076] 
21. Hu A, Wang F, Sellers JR. Mutations in human nonmuscle myosin IIA found in patients with May-Hegglin anomaly and Fechtner syndrome result in impaired enzymatic function. J. Biol. Chem. 2002; 277:46512-46517. [PubMed: 12237319]

22. Kim KY, Kovacs M, Kawamoto S, Sellers JR, Adelstein RS. Disease-associated mutations and alternative splicing alter the enzymatic and motile activity of nonmuscle myosins II-B and II.-C. J. Biol. Chem. 2005; 280:22769-22775. [PubMed: 15845534]

23. Otani T, Ichii T, Aono S, Takeichi M. Cdc42 GEF Tuba regulates the junctional configuration of simple epithelial cells. J. Cell Biol. 2006; 175:135-146. [PubMed: 17015620]

24. Yap AS, Brieher WM, Pruschy M, Gumbiner BM. Lateral clustering of the adhesive ectodomain: a fundamental determinant of cadherin function. Curr. Biol. 1997; 7:308-315. [PubMed: 9133345]

25. McLachlan RW, Kraemer A, Helwani FM, Kovacs EM, Yap AS. E-cadherin adhesion activates cSrc signaling at cell-cell contacts. Mol. Biol. Cell. 2007; 18:3214-3223. [PubMed: 17553930]

26. Yap AS, Niessen C, Gumbiner BM. The juxtamembrane region of the cadherin cytoplasmic tail supports lateral clustering, adhesive strengthening and interaction with p120 $\mathrm{ctn}$. J. Cell Biol. 1998; 141:779-789. [PubMed: 9566976]

27. Gavard J, et al. Lamellipodium extension and cadherin adhesion: two cell responses to cadherin activation relying on distinct signalling pathways. J. Cell Sci. 2004; 117:257-270. [PubMed: 14657280]

28. Scott JA, et al. Ena/VASP proteins can regulate distinct modes of actin organization at cadherinadhesive contacts. Mol. Biol. Cell. 2006; 17:1085-1095. [PubMed: 16371509]

29. Meng W, Mushika Y, Ichii T, Takeichi M. Anchorage of microtubule minus ends to adherens junctions regulates epithelial cell-cell contacts. Cell. 2008; 135:948-959. [PubMed: 19041755]

30. Kametani Y, Takeichi M. Basal-to-apical cadherin flow at cell junctions. Nat. Cell Biol. 2007; 9:92-98. [PubMed: 17159998]

\section{References}

31. Goodwin M, Kovacs EM, Thoreson MA, Reynolds AB, Yap AS. Minimal mutation of the cytoplasmic tail inhibits the ability of E-cadherin to activate Rac but not phosphatidylinositol 3kinase: direct evidence of a role for cadherin-activated Rac signaling in adhesion and contact formation. J. Biol. Chem. 2003; 278:20533-20539. [PubMed: 12672818]

32. Helwani FM, et al. Cortactin is necessary for E-cadherin-mediated contact formation and actin reorganization. J. Cell Biol. 2004; 164:899-910. [PubMed: 15024035]

33. Kovacs EM, Ali RG, McCormack AJ, Yap AS. E-cadherin homophilic ligation directly signals through Rac and phosphatidylinositol 3-kinase to regulate adhesive contacts. J. Biol. Chem. 2002; 277:6708-6718. [PubMed: 11744701]

34. Verma $S$, et al. Arp2/3 activity is necessary for efficient formation of E-cadherin adhesive contacts. J. Biol. Chem. 2004; 279:34062-34070. [PubMed: 15159390]

35. Brummelkamp TR, Bernards R, Agami R. A system for stable expression of short interfering RNAs in mammalian cells. Science. 2002; 296:550-553. [PubMed: 11910072]

36. Rubinson DA, et al. A lentivirus-based system to functionally silence genes in primary mammalian cells, stem cells and transgenic mice by RNA interference. Nature Genet. 2003; 33:401-406. [PubMed: 12590264]

37. Vitriol EA, Uetrecht AC, Shen F, Jacobson K, Bear JE. Enhanced EGFP-chromophore-assisted laser inactivation using deficient cells rescued with functional EGFP-fusion proteins. Proc. Natl Acad. Sci. USA. 2007; 104:6702-6707. [PubMed: 17420475] 
a
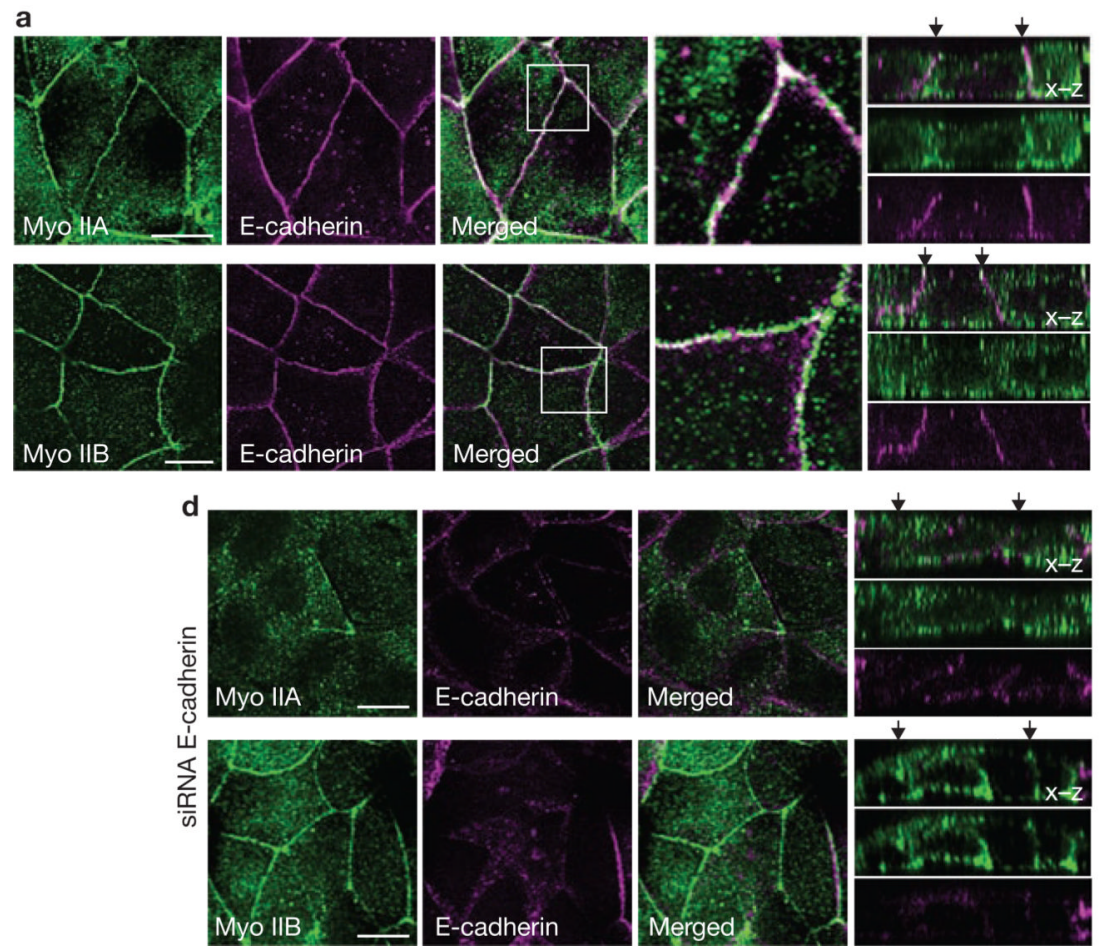

b

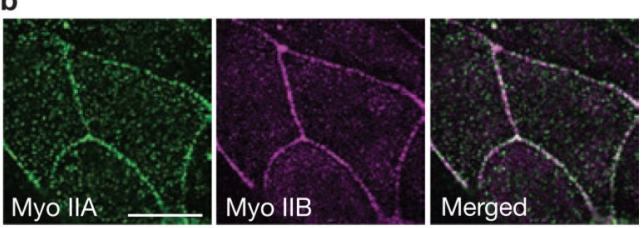

c
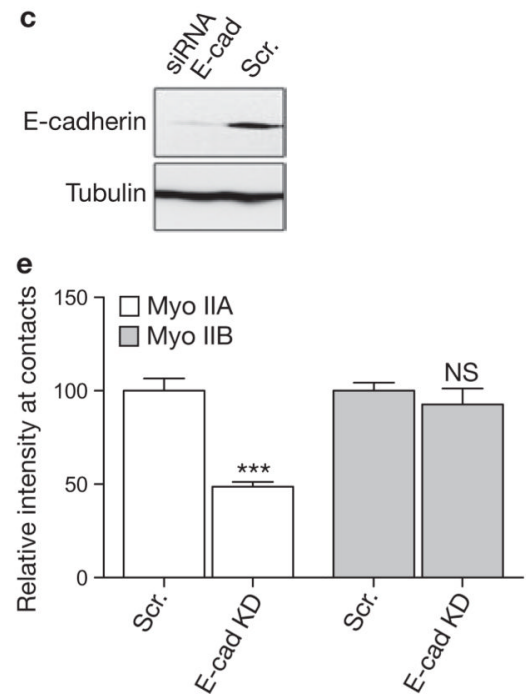

Figure 1. Myosin (Myo) IIA and myosin IIB localize to apical epithelial junctions

(a) Confluent MCF-7 cells were fixed and immunostained for E-cadherin (magenta) and for myosin IIA (green) or myosin IIB (green). Representative confocal images were taken from the apical junctions of the cells. Magnifications show detailed colocalization of E-cadherin with myosin II isoforms and the distribution of proteins along the $z$ axis of cells are represented in $X-Z$ views (where apical is up). Arrows indicate enrichment of E-cadherin and myosin II at the apical tips of cell-cell contacts. (b) Confocal images show co-localization of myosin IIA (green) and myosin IIB (magenta) at apical junctions in MCF-7 monolayers. (c) E-cadherin RNAi. MCF-7 cells were transfected with siRNA against E-cadherin or scrambled (scr.) siRNA; E-cadherin levels were assessed by immunoblot analysis $48 \mathrm{~h}$ after transfection. Tubulin was used as a loading control. (d) E-cadherin KD cells were fixed $48 \mathrm{~h}$ after transfection and immunostained for E-cadherin and either myosin IIA or myosin IIB; $x-y$ images in the apical plane and $x-Z$ reconstructions are shown. Arrows indicate apical regions of cell-cell contacts. (e) Junctional accumulation of myosin II isoforms was quantified by measuring fluorescence intensity at cell-cell contacts in control and Ecadherin KD cells. Data are represented as means \pm s.e.m. $(n=21$; three asterisks, $P<$ 0.001; Student's $t$-test). Scale bars, $10 \mu \mathrm{m}$. 


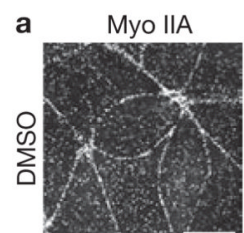

E-cadherin
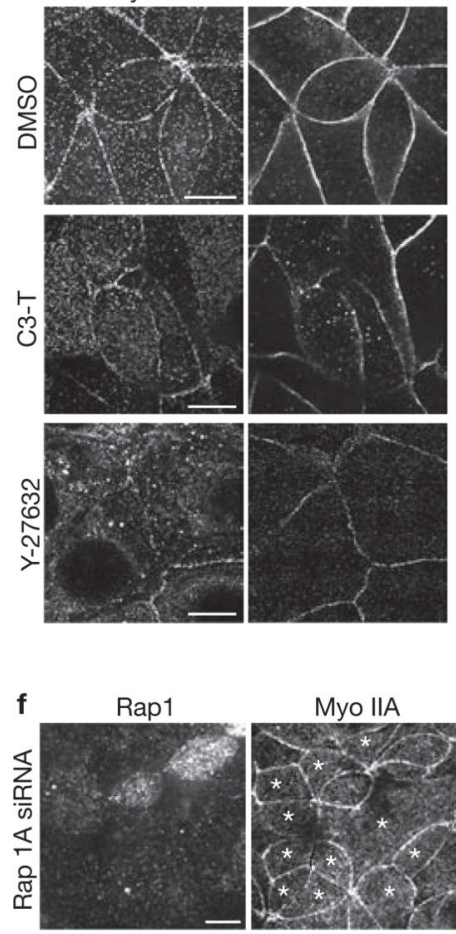

Myo IIA
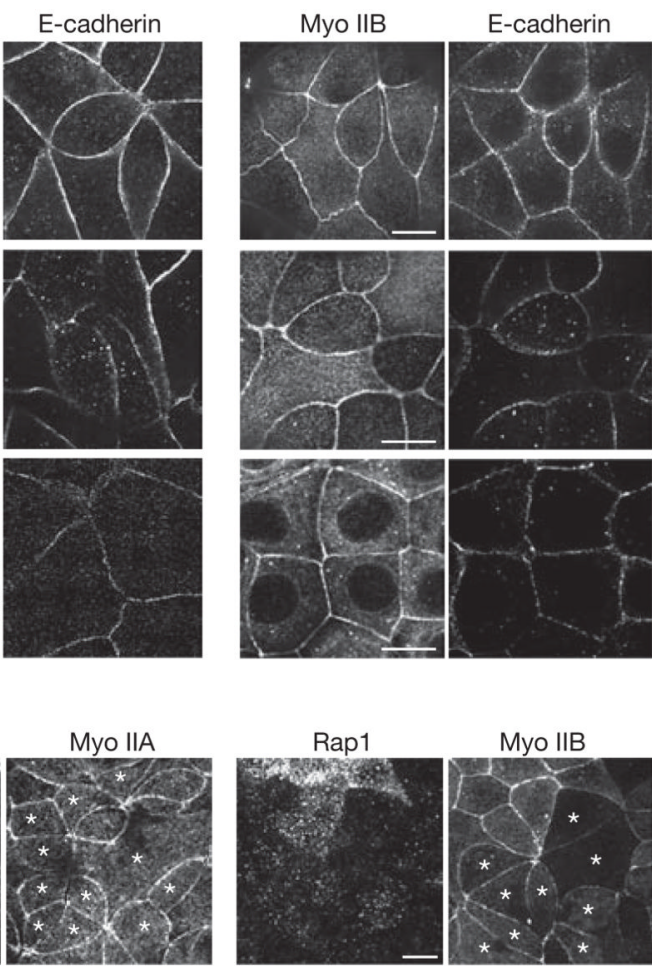
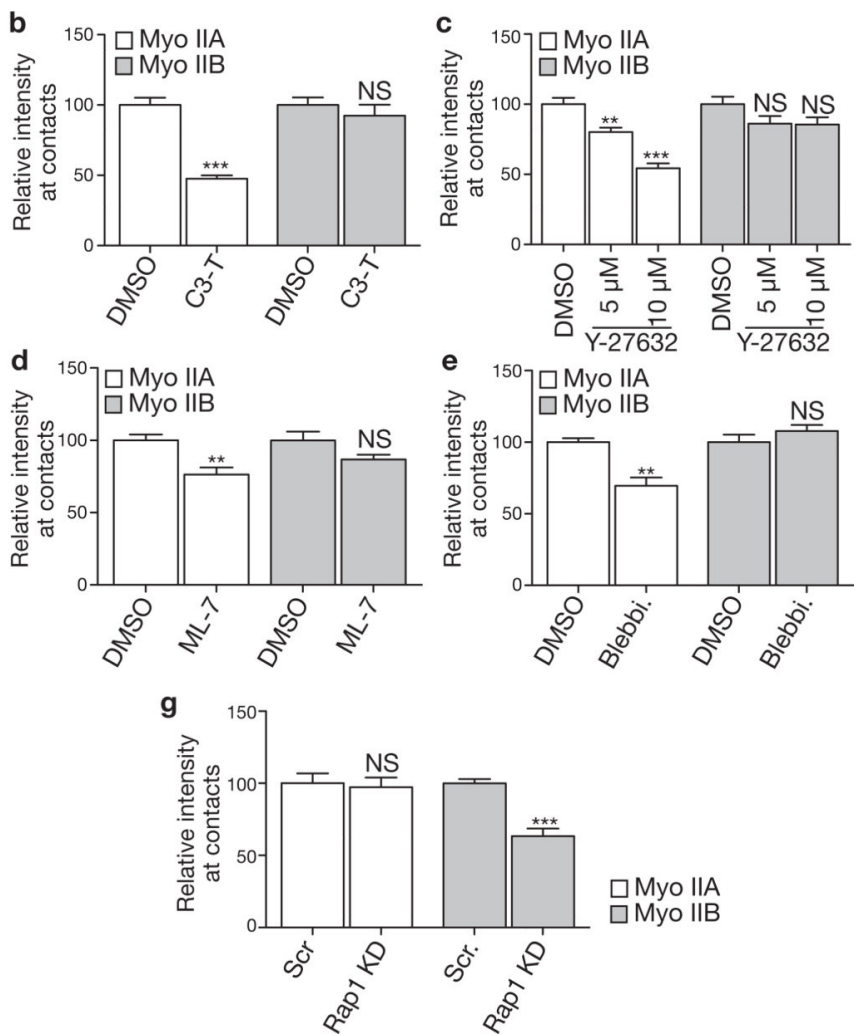

Figure 2. Differential regulation of myosin IIA and myosin IIB localization at apical junctions (a-c) Impact of Rho and ROCK on junctional localization of myosin II isoforms. MCF-7 monolayers were incubated with Y-27632 (5-10 $\mu \mathrm{M})$, the Rho inhibitor C3-transferase (C3$\mathrm{T}, 0.5 \mu \mathrm{g} \mathrm{ml}^{-1}$ ) or dimethylsulphoxide (DMSO) for $3 \mathrm{~h}$, then fixed and immunostained for E-cadherin and myosin IIA or myosin IIB. (a) Representative apical confocal images. (b, c) Junctional localization of myosin IIA or myosin IIB was quantified by fluorescence intensity analysis of contacts in cells treated with C3-T (b) or Y-27632 (c). Data are means and s.e.m. $(n=24)$. (d) MLCK supports junctional localization of myosin IIA but not that of myosin IIB. Myosin isoform localization at junctions was quantified in cells treated with ML7 (10 $\mu \mathrm{M}, 3 \mathrm{~h})$. Data are represented as means \pm s.e.m. $(n=24)$. (e) Myosin activity is necessary for junctional localization of myosin IIA but not that of myosin IIB. Junctional localization of myosin II isoforms was measured in cells treated with blebbistatin (blebbi.) $(100 \mu \mathrm{M}, 3$ h). Data are represented as means \pm s.e.m. $(n=24)$. (f, $\mathbf{g})$ Rap1 supports junctional localization of myosin IIB. MCF-7 cells were transfected with siRNA against Rap 1A (100 $\mathrm{nM})$ or scrambled siRNA $(100 \mathrm{nM})$. (f) After $48 \mathrm{~h}$ cells were fixed and immunostained for Rap 1 and either myosin IIA or myosin IIB. Transfected cells that show reduced levels of Rap1 are identified with asterisks in the myosin staining. (g) Junctional localization of myosin isoforms was measured by fluorescence intensity analysis. Data are represented as means \pm s.e.m. $(n=21)$. For all experiments: two asterisks, $P<0.01$; three asterisks, $P<$ 0.001; Student's $t$-test. Scale bars, $10 \mu \mathrm{m}$. 
a
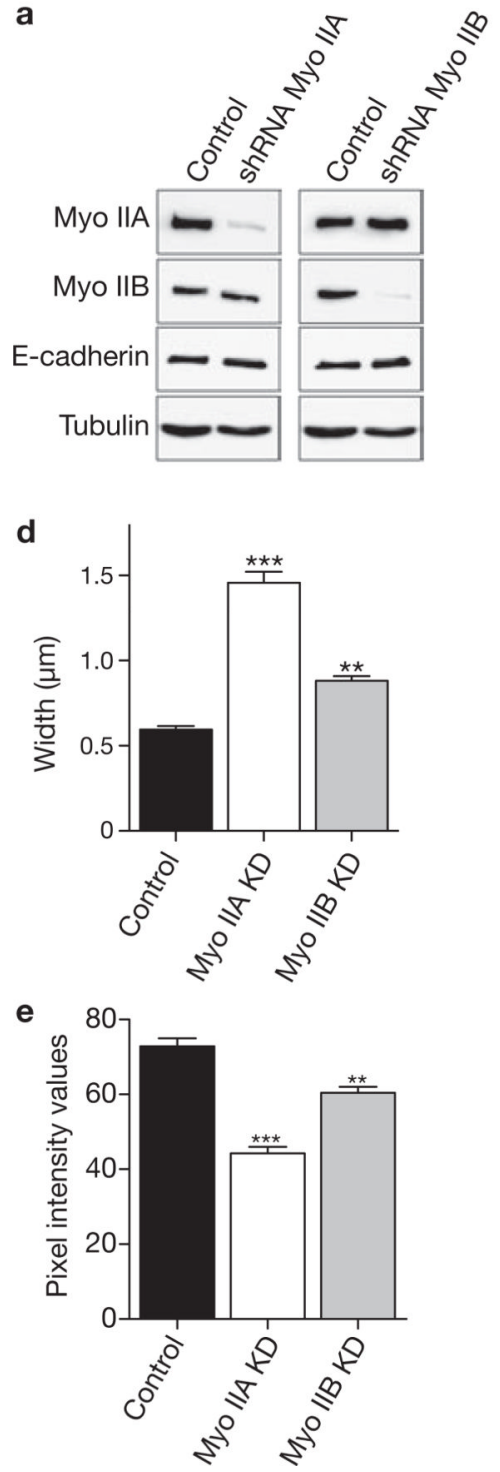

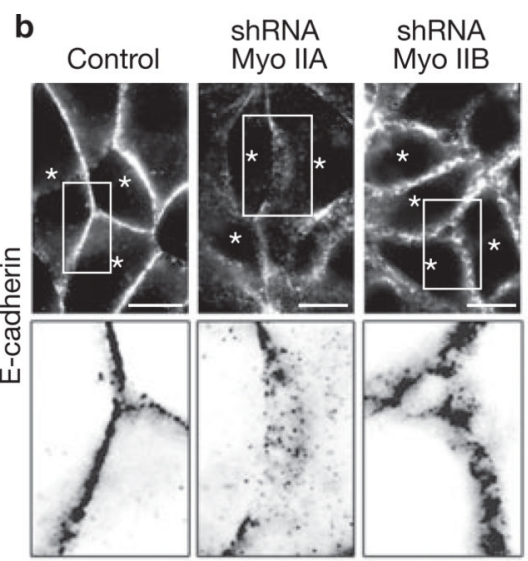

f
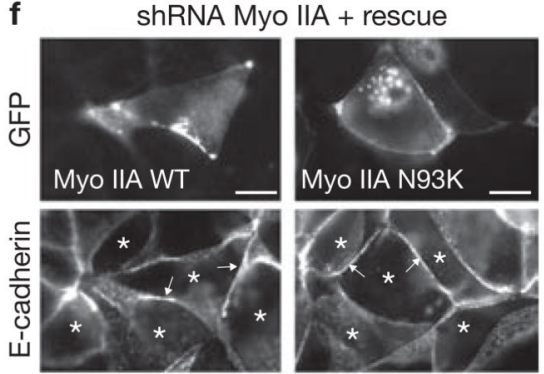

g

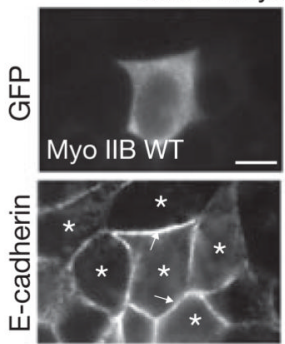

C
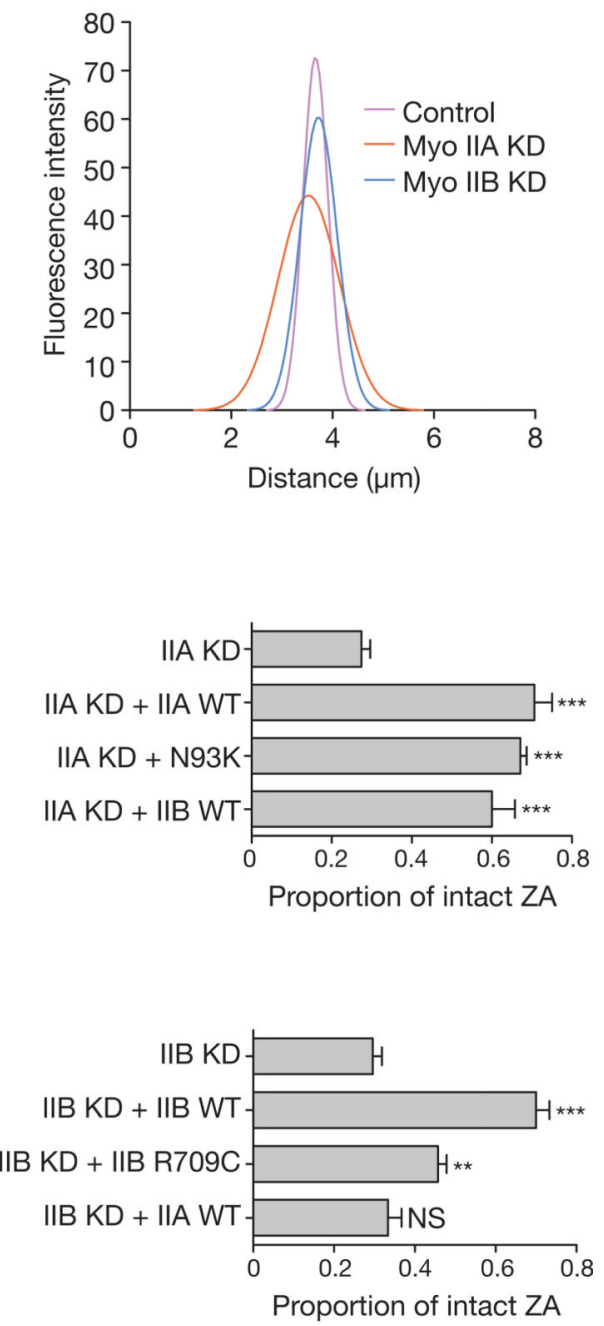

Figure 3. Myosin IIA and myosin IIB are necessary for ZA integrity (a) Isoform-specific myosin II depletion. MCF-7 cells were infected with lentivirus bearing shRNA directed against either myosin IIA or myosin IIB. Control cells were infected with virus coding for soluble fluorophores alone. Selective depletion of the appropriate myosin II isoform was confirmed by immunoblot analysis 10-15 days after infection. Lysates were also probed for E-cadherin and tubulin as a loading control. (b) E-cadherin morphology at cell-cell contacts was assessed by immunolabelling for E-cadherin in shRNA and control cells. Transduced cells are marked with asterisks. Contrast is inverted in the detail areas to highlight the different impact of each myosin II isoform KD on E-cadherin morphology. (ce) Changes in E-cadherin morphology at cell contacts were quantified by line scan analysis of fluorescence intensity. (c) Nonlinear fit curves of contact profiles in control and myosin isoform KD cells $(n=24)$. Means and s.e.m. of contact profiles are shown in Supplementary Information, Fig. S4c. (d) The lateral distribution of E-cadherin at cell-cell contacts was measured by calculating the average of the full width at half-maximum for each contact profile. (e) Peak E-cadherin fluorescence intensity in myosin isoform KD and control cells. $(\mathbf{f}, \mathbf{g})$ Rescue of junctional integrity in shRNA cells by reconstitution with wild-type or 
mutant myosin II transgenes. GFP-tagged, RNAi-resistant wild-type (WT) or N93K myosin IIA (f) and WT or R709C myosin IIB (g) transgenes were transiently expressed in the appropriate isoform KD cells. WT myosin IIB was also expressed in myosin IIA KD cells (f), whereas WT myosin IIA was expressed in myosin IIB KD cells (g). Cells were stained for E-cadherin and scored for restoration of an intact ZA. Representative images and quantification are shown $(n=90)$. Asterisks mark KD cells (identified by transduced mCherry staining, not shown). For all experiments: two asterisks, $P<0.01$; three asterisks, $P$ $<0.001$; analysis of variance with Dunnett's test. Scale bars, $10 \mu \mathrm{m}$. 


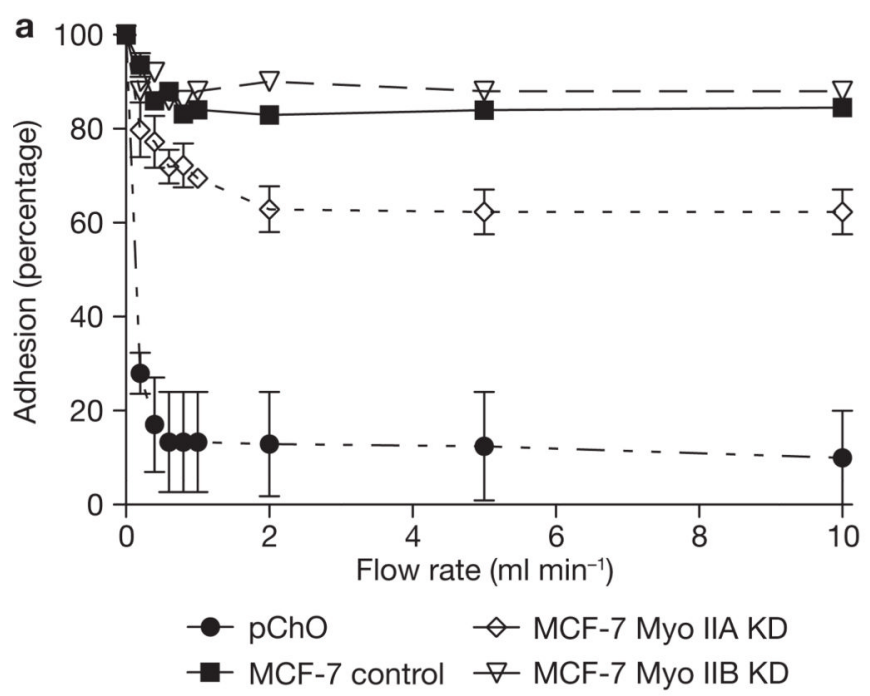

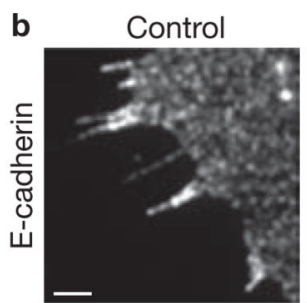

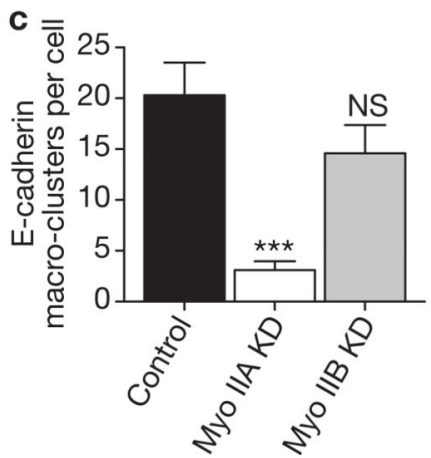

Myo IIA KD

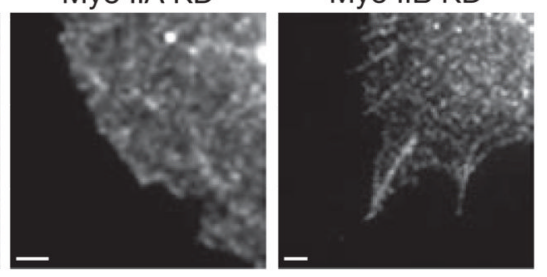

d $\stackrel{0}{5}$

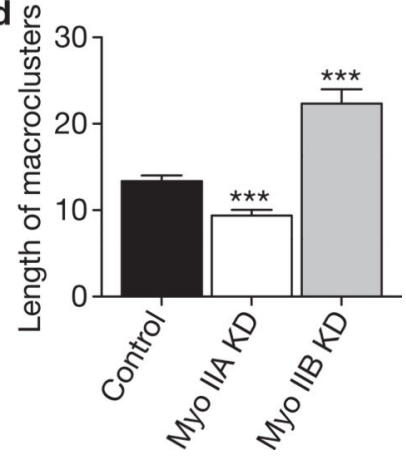

Figure 4. Homophilic adhesion and lateral clustering of E-cadherin requires myosin IIA but not myosin IIB

(a) Homophilic adhesion of E-cadherin to cadherin-coated substrata was measured for control and myosin isoform KD MCF-7 cells. Adhesion in laminar-flow assays was expressed as the percentage of cells that remained adherent to $\mathrm{hE} / \mathrm{Fc}$-coated substrata at increasing flow rates. E-cadherin-deficient $\mathrm{CHO}(\mathrm{pCHO})$ cells were used as negative controls. Data are represented as means \pm s.e.m. ( $n=3$ independent experiments). (b-d) Lateral cadherin clustering was assessed in planar adhesion assays. Control MCF-7 cells, or Myo IIA and Myo IIB KD cells, were allowed to spread onto hE/Fc-coated substrata for 70 min; they were then fixed and stained for cellular E-cadherin to identify streak-like cadherin macroclusters (b). Images represent regions of the cell interface with $\mathrm{hE} / \mathrm{Fc}$-coated substrata revealed by confocal microscopy. Scale bars, $1 \mu \mathrm{m}$. (c) E-cadherin macroclusters were quantified by counting the number of macroclusters in myosin II isoform KD and control cells. Data are represented as means \pm s.e.m. $(n=10)$. (d) E-cadherin macrocluster length was quantified by measuring the number of pixels along each cluster in control and KD cells. Data are represented as means \pm s.e.m. $(n=60)$. Two asterisks, $P<0.01$; Student's $t$ test. 
a
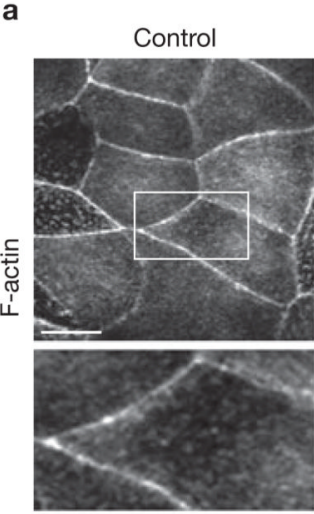
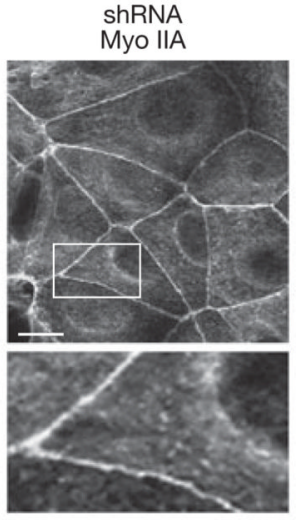

d

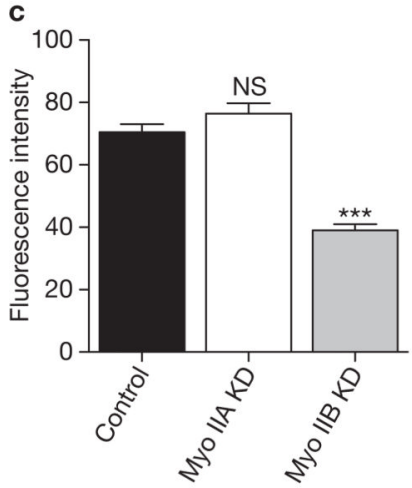

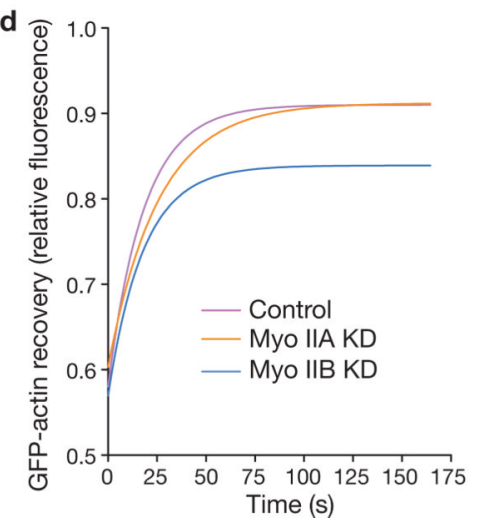
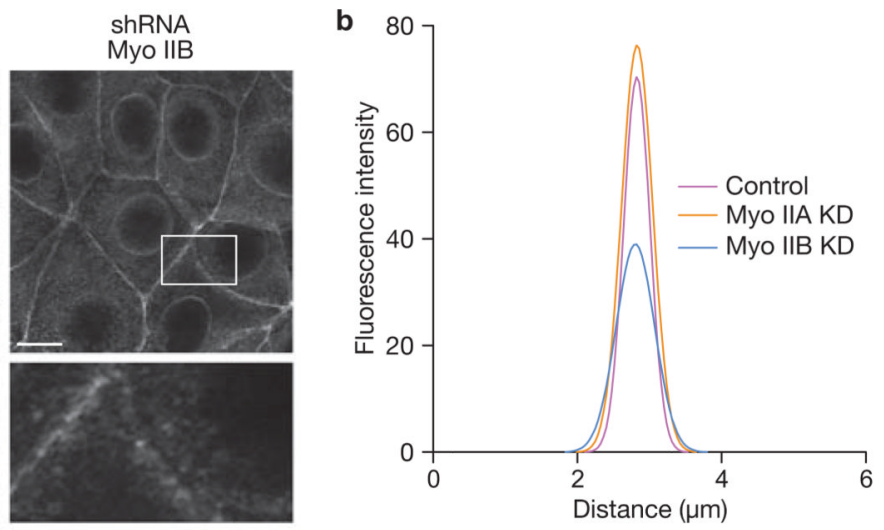

Figure 5. Myosin IIB regulates the apical F-actin ring

(a) Representative confocal images of the perijunctional F-actin ring in control and myosin II isoform KD cells identified by phalloidin staining. Detailed views of F-actin at cell-cell contacts are shown below in magnifications. Scale bars, $10 \mu \mathrm{m}$. (b-c) F-actin content in the perijunctional rings was quantified by line scan profile analysis of fluorescence intensity at contacts between control and myosin isoform KD cells. (b) Nonlinear fit curves of pooled intensity profiles $(n=24)$. Intensity profiles (means and s.e.m.) are shown in Supplementary Information, Fig. S6a. (c) Average peak fluorescence intensity values of actin in knockdown and control cells. Data are means and s.e.m. $(n=24$; three asterisks, $P<0.001$; Student's $t$ test). (d) Junctional actin turnover in control or myosin isoform KD MCF-7 cells.

Transiently transfected GFP-actin was revealed by live-cell imaging, and GFP-actin in the apical actin ring was bleached in a region of interest. FRAP recovery is represented by curve fits of GFP-actin fluorescence $(n=8)$; average fluorescence intensity profiles ( \pm s.e.m.) are shown in Supplementary Information, Fig. S6b. (e) Lateral movement of the apical actin ring. Kymographs from photobleaching data were performed by using line scans perpendicular to the GFP-actin ring. Representative kymographs, an illustration of the quantification method, and scatter plots of lateral movement are shown $(n=8$; two asterisks, $P<0.001$; analysis of variance with Dunnett's test). Lateral movement of the ring away from the initial position (red line) was calculated by measuring the angle between the initial position and the final position in either direction (blue dotted lines show the extremes of angles). 